\title{
Leukocyte subtypes and adverse clinical outcomes in patients with acute ischemic cerebrovascular events
}

\author{
Anxin Wang ${ }^{1,2 \#}$, Kehua Quan ${ }^{1,2 \#}$, Xue Tian ${ }^{1,2}$, Yingting Zuo ${ }^{1,2}$, Xia Meng ${ }^{1,2}$, Pan Chen ${ }^{1,2}$, Hao Li ${ }^{1,2}$, \\ Yongjun Wang ${ }^{1,2}$ \\ ${ }^{1}$ China National Clinical Research Center for Neurological Diseases, Beijing Tiantan Hospital, Capital Medical University, Beijing, China; \\ ${ }^{2}$ Department of Neurology, Beijing Tiantan Hospital, Capital Medical University, Beijing, China \\ Contributions: (I) Conception and design: A Wang, K Quan, Y Wang; (II) Administrative support: X Meng, P Chen, H Li, Y Wang; (III) Provision \\ of study materials or patients: A Wang, X Meng, P Chen, H Li, Y Wang; (IV) Collection and assembly of data: K Quan, X Tian, Y Zuo; (V) Data \\ analysis and interpretation: A Wang, K Quan, X Tian, Y Zuo; (VI) Manuscript writing: All authors; (VII) Final approval of manuscript: All authors. \\ \#These authors contributed equally to this work. \\ Correspondence to: Yongjun Wang. Department of Neurology, Beijing Tiantan Hospital, Capital Medical University, No. 119 South 4th Ring West \\ Road, Fengtai District, Beijing 100070, China. Email: yongjunwang@ncrend.org.cn.
}

\begin{abstract}
Background: Our study aimed to evaluate whether the effects on adverse clinical outcomes, defined as death, recurrent stroke, and poor functional outcomes, differed by leukocyte subtype in patients with acute ischemic cerebrovascular events, including both ischemic stroke and transient ischemic attack (TIA).

Methods: We derived data from the Third China National Stroke Registry (CNSR-III). The counts and percentages of each leukocyte subtype were collected within the first 24 hours after admission. Enrolled patients were classified into four groups by the quartiles of each leukocyte subtype count or percentage. Hazard ratios (HRs) or odds ratios (ORs) and their 95\% confidence intervals (CIs) of adverse clinical outcomes were calculated, with the lowest quartile group as the reference category. We used C statistics, integrated discrimination improvement (IDI), and the net reclassification index (NRI) to evaluate each leukocyte subtype's incremental predictive value beyond conventional risk factors.

Results: A total of 14,174 patients were enrolled. Higher counts of leukocytes, neutrophils, and monocytes were associated with elevated risks of adverse clinical outcomes. In contrast, higher counts of lymphocytes and eosinophils were related to reduced risks of adverse clinical outcomes. Meanwhile, basophil counts seemed to not correlate with adverse clinical outcomes. Furthermore, there were also significant associations between the percentages of leukocyte subtypes and adverse clinical outcomes.

Conclusions: Leukocyte subtypes had different relationships with adverse clinical outcomes at 3-month and 1-year follow-up in patients with acute ischemic cerebrovascular events and could slightly increase the predictive value compared with the conventional model.
\end{abstract}

Keywords: Leukocyte; ischemic stroke; transient ischemic attack (TIA); outcome

Submitted Dec 10, 2020. Accepted for publication Mar 10, 2021.

doi: 10.21037/atm-20-7931

View this article at: http://dx.doi.org/10.21037/atm-20-7931

\section{Introduction}

The peripheral immune system can respond robustly to acute ischemic stroke (1). It is increasingly thought that the activation and variations in circulating immune cells' levels are among the early biological responses detected after acute ischemic stroke $(2,3)$. In turn, the inflammation mediated by circulating immune cells has been identified as one of the key elements in the subsequent clinical course of acute ischemic stroke, and recent studies suggest that it can influence brain injury and clinical outcomes (4). One of the most notable inflammation responses after acute ischemic stroke is the leukocyte count change (5). Data consistently 
show that acute ischemic stroke can induce leukocytosis, which has been proven to be associated with increased mortality, disability at discharge, early-onset post-stroke delirium, and prolonged hospitalization (4,6-9).

Similarly, our previous study also concluded that leukocyte count at admission was correlated with both short- and long-term clinical outcomes in acute ischemic stroke patients and might have a role as a poor prognostic factor (10). It is well known that leukocytes can be divided into neutrophils, lymphocytes, monocytes, eosinophils, and basophils. After ischemic stroke, the temporal changes in the levels of leukocyte subtypes are varied (4). Moreover, each leukocyte subtype has different immunological functions and contributes differently to the pathophysiology of atherosclerosis and cerebrovascular diseases $(11,12)$. Therefore, leukocytes' effects on clinical outcomes in patients with acute ischemic stroke may vary depending on different subtypes, which has been revealed in several studies $(3,12)$. However, generally, these studies still have some limitations, such as small sample sizes, short followup times, and less reporting on the outcome of stroke recurrence. Also, as far as we know, few studies have focused on the relationship between leukocytes and the clinical outcomes of transient ischemic attack (TIA) patients. Our study aimed to evaluate whether the effects on adverse clinical outcomes, defined as death, recurrent stroke, and poor functional outcomes, differed by leukocyte subtype in patients with acute ischemic cerebrovascular events, including both ischemic stroke and TIA. We present the following article in accordance with the STROBE reporting checklist (available at http://dx.doi.org/10.21037/atm-20-7931).

\section{Methods}

\section{Study design and population}

We derived data from the Third China National Stroke Registry (CNSR-III). The CNSR-III is a large-scale nationwide, multicenter, prospective clinical registry study of patients with acute ischemic cerebrovascular events who presented to hospitals between August 2015 and March 2018 in China. Details of the study design and major results have been described previously (13). Briefly, 15,166 patients were recruited consecutively from 201 hospitals who met the following criteria: (I) age older than 18 years; (II) diagnosis of ischemic stroke or TIA; (III) within 7 days from the onset of symptoms to enrollment; and (IV) informed consent from the patient or legally authorized representative. Acute ischemic stroke was diagnosed according to the WHO criteria and confirmed by MRI or brain CT. Among the enrolled patients in the CNSRIII, we excluded 992 patients without available complete blood counts on admission or who were lost to follow-up (Figure S1). The ethics committee at Beijing Tiantan Hospital (IRB approval number: KY2015-001-01) and all study centers gave the CNSR-III study protocol ethical approval. All patients or their legal representatives provided written informed consent before being entered into the CNSR-III study. This study was conducted following the Declaration of Helsinki (as revised in 2013).

\section{Data collection and calculation}

Trained neurologists at each participating hospital systematically collected baseline data, including age, sex, body mass index (BMI), smoking and drinking status, medical history, National Institutes of Health Stroke Scale (NIHSS) score at admission, time from symptom onset to enrollment, and therapy, through face-to-face interviews or medical records.

Fasting whole blood samples from venipuncture were collected in vacutainer tubes containing EDTA within the first 24 hours after admission and kept at room temperature. Afterward, the count of each leukocyte subtype was analyzed by an automated hematology analyzer at each participating hospital. All measurements were performed by laboratory personnel blinded to patients' clinical situations. Each leukocyte subtype percentage was calculated as the ratio of its absolute count to total leukocyte count.

\section{Outcome assessment}

Patients were followed up by face-to-face interviews at 3 months and contacted over the telephone at 1 year by trained research coordinators. Information including functional status and cerebrovascular events were queried at each follow-up. Any all-cause death and stroke recurrence during the follow-up periods were recorded. The fatality was either confirmed on a death certificate from the attended hospital or the local civil registry. Recurrent stroke included both ischemic and hemorrhagic stroke, which was confirmed from the treating hospital, and suspected events without hospitalization were judged by an independent endpoint judgment committee. The modified Rankin Scale (mRS) was used to assess patients' functional dependence, and poor functional outcomes were defined as $3 \leq \mathrm{mRS} \leq 5$. 


\section{Statistical analysis}

Continuous variables were described as medians with interquartile ranges (IQRs) and categorical variables as percentages. Baseline characteristics were compared across the quartile groups of leukocyte counts using the chisquare test or Fisher's exact test for categorical variables and the Kruskal-Wallis test for continuous variables. Enrolled patients were classified into four groups by the quartiles of each leukocyte subtype count or percentage. The Cox proportional hazards regression model was used to investigate each leukocyte subtype's associations with death and stroke recurrence. The logistic regression model was used to assess the correlations between each leukocyte subtype and poor functional outcomes. To adjust for other potential confounding variables, multivariable analyses including age, sex, BMI, drinking, smoking, hypertension, lipid metabolism disorders, diabetes, previous stroke, atrial fibrillation, heart failure, heart valve disease, peripheral arterial disease, infection within 2 weeks before admission, intravenous thrombolysis, endovascular therapy, time from symptom onset to enrollment, and the NIHSS score at admission were performed. Crude and adjusted hazard ratios (HR) or odds ratios (ORs) and their $95 \%$ confidence intervals (CIs) were calculated with the lowest quartile group as the reference category. We further evaluated the pattern of correlation between each leukocyte subtype count on a continuous scale and the risk of adverse clinical outcomes at 1-year follow-up using a multivariable Cox regression model of the restricted cubic spline with adjustment for potential covariates. Also, we used $\mathrm{C}$ statistics, integrated discrimination improvement (IDI) and net reclassification index (NRI) to evaluate the incremental predictive value of each leukocyte subtype beyond conventional risk factors, which included age, sex, BMI, smoking, drinking, hypertension, previous stroke, lipid metabolism disorders, diabetes, atrial fibrillation, coronary heart disease, peripheral arterial disease, heart failure, heart valve disease, NIHSS at admission, time from symptom onset to enrollment, intravenous thrombolysis, endovascular therapy, antiplatelet agents, anticoagulant drugs, lipid-lowering drugs, hypoglycemic treatment, and antihypertensive treatment. Overall, a two-sided $\mathrm{P}$ value of $<0.05$ was considered statistically significant. All statistical analyses were performed with SAS software version 9.4 (SAS Institute Inc., Cary, NC, USA).

\section{Results}

\section{Baseline characteristics}

A total of 14,174 patients were included in our analysis. Table S1 showed that included and excluded patients' baseline characteristics were well balanced, except the included patients were younger and had a higher proportion of lipid metabolism disorder, lower NIHSS scores at admission, and lower proportions of hypertension and intravenous thrombolysis.

The baseline characteristics of the included patients stratified according to quartiles of leukocyte counts are shown in Table 1. Compared to the patients with a lower leukocyte count, those in the higher quartile groups were more likely to be smoking males with lower NIHSS scores at admission, and a lower proportion of these patients received endovascular therapy and intravenous thrombolysis. In patients with a higher leukocyte count, atrial fibrillation and infection within 2 weeks before admission were less frequent, while lipid metabolism disorder was more frequent. Moreover, there were differences in age and time from symptom onset to enrollment among patients in the leukocyte count quartile groups.

\section{Leukocyte subtypes and adverse clinical outcomes}

The risks of adverse clinical outcomes at 1-year follow-up in the quartile groups of each leukocyte subtype count are shown in Figure 1 and Table S2. Higher leukocyte count and neutrophil count were obviously related to elevated risks of death, stroke recurrence, and poor functional outcomes at 1-year follow-up compared with the lowest quartile group taken as the reference. Similar associations were also found between monocyte count and both death and poor functional outcomes at 1-year follow-up. The above relationships were still significant after adjustments. Conversely, higher lymphocyte and eosinophil counts were associated with reduced risks of death, stroke recurrence, and poor functional outcomes at 1-year follow-up. However, after adjustments, lymphocyte count only had a significant association with poor functional outcomes, and the relationship between eosinophil count and death no longer reached statistical significance. There were no significant correlations between basophil count and death, stroke recurrence, or poor functional outcomes at 1-year follow-up. Multivariable-adjusted spline regression models 
Table 1 Baseline characteristics of included patients stratified by leukocyte count

\begin{tabular}{|c|c|c|c|c|c|}
\hline Baseline characteristics & Q1 & Q2 & Q3 & Q4 & $\mathrm{P}$ \\
\hline Age, median [IQR], y & $62[54-70]$ & $63[55-71]$ & $63[54-70]$ & 62 [54-69] & 0.0056 \\
\hline Female, n (\%) & $1,151(32.50)$ & $1,201(33.95)$ & $1,120(31.54)$ & $1,021(28.82)$ & $<0.0001$ \\
\hline $\mathrm{BMI}, \mathrm{kg} / \mathrm{m}^{2}$, median $[\mathrm{IQR}]$ & 24.49 [22.58-26.44] & $\begin{array}{l}24.49[22.49- \\
26.57]\end{array}$ & 24.54 [22.68-26.67] & $24.49[22.6-26.57]$ & 0.3458 \\
\hline Current drinking, n (\%) & $562(15.87)$ & $541(15.29)$ & $578(16.28)$ & $619(17.47)$ & 0.0836 \\
\hline \multicolumn{6}{|l|}{ Medical history, n (\%) } \\
\hline Previous stroke & 787 (22.22) & $802(22.67)$ & $780(21.97)$ & 779 (21.99) & 0.8838 \\
\hline Atrial fibrillation & $269(7.59)$ & $247(6.98)$ & $223(6.28)$ & $204(5.76)$ & 0.0115 \\
\hline Heart failure & $24(0.68)$ & $22(0.62)$ & $23(0.65)$ & $17(0.48)$ & 0.7165 \\
\hline Heart valve disease & $13(0.37)$ & $14(0.40)$ & $14(0.39)$ & $13(0.37)$ & 0.9948 \\
\hline Hypertension & $2,199(62.08)$ & $2,218(62.69)$ & $2,225(62.66)$ & $2,201(62.12)$ & 0.9199 \\
\hline Lipid metabolism disorder & $239(6.75)$ & $287(8.11)$ & $287(8.08)$ & $323(9.12)$ & 0.0035 \\
\hline Peripheral arterial disease & $32(0.90)$ & $22(0.62)$ & $32(0.90)$ & $24(0.68)$ & 0.3902 \\
\hline $\begin{array}{l}\text { Infection within } 2 \text { weeks before } \\
\text { admission }\end{array}$ & $123(3.47)$ & $127(3.59)$ & $95(2.68)$ & $80(2.26)$ & 0.0019 \\
\hline \multicolumn{6}{|l|}{ Therapy, n (\%) } \\
\hline Intravenous thrombolysis & $365(10.30)$ & $409(11.56)$ & $342(9.63)$ & $288(8.13)$ & $<0.0001$ \\
\hline Endovascular therapy & $35(0.99)$ & $22(0.62)$ & $20(0.56)$ & $11(0.31)$ & 0.0037 \\
\hline
\end{tabular}

IQR, interquartile range; BMI, body mass index; TIA, transient ischemic attack; NIHSS, National Institutes of Health Stroke Scale.

for associations between each leukocyte subtype count and adverse clinical outcomes at 1 -year follow-up are shown in Figure 2.

The above relationships between each leukocyte subtype count and risks of adverse clinical outcomes were still valid at 3-month follow-up, as shown in Table S3. However, eosinophil count did not have a significant association with stroke recurrence at 3-month follow-up after adjustments. Furthermore, a higher basophil count was correlated with a reduced risk of poor functional outcomes at 3-month follow-up only before adjustments.

Also, we assessed the risks of adverse clinical outcomes at 1-year follow-up in quartile groups of each leukocyte subtype percentage, which are shown in Figure 3 and Table S4. Patients with a higher neutrophil percentage showed higher incidences of death, stroke recurrence, and poor functional outcomes at 1-year follow-up. In contrast, lower risks of death, stroke recurrence, and poor functional outcomes at 1-year follow-up were found in patients with a higher lymphocyte percentage and eosinophil 


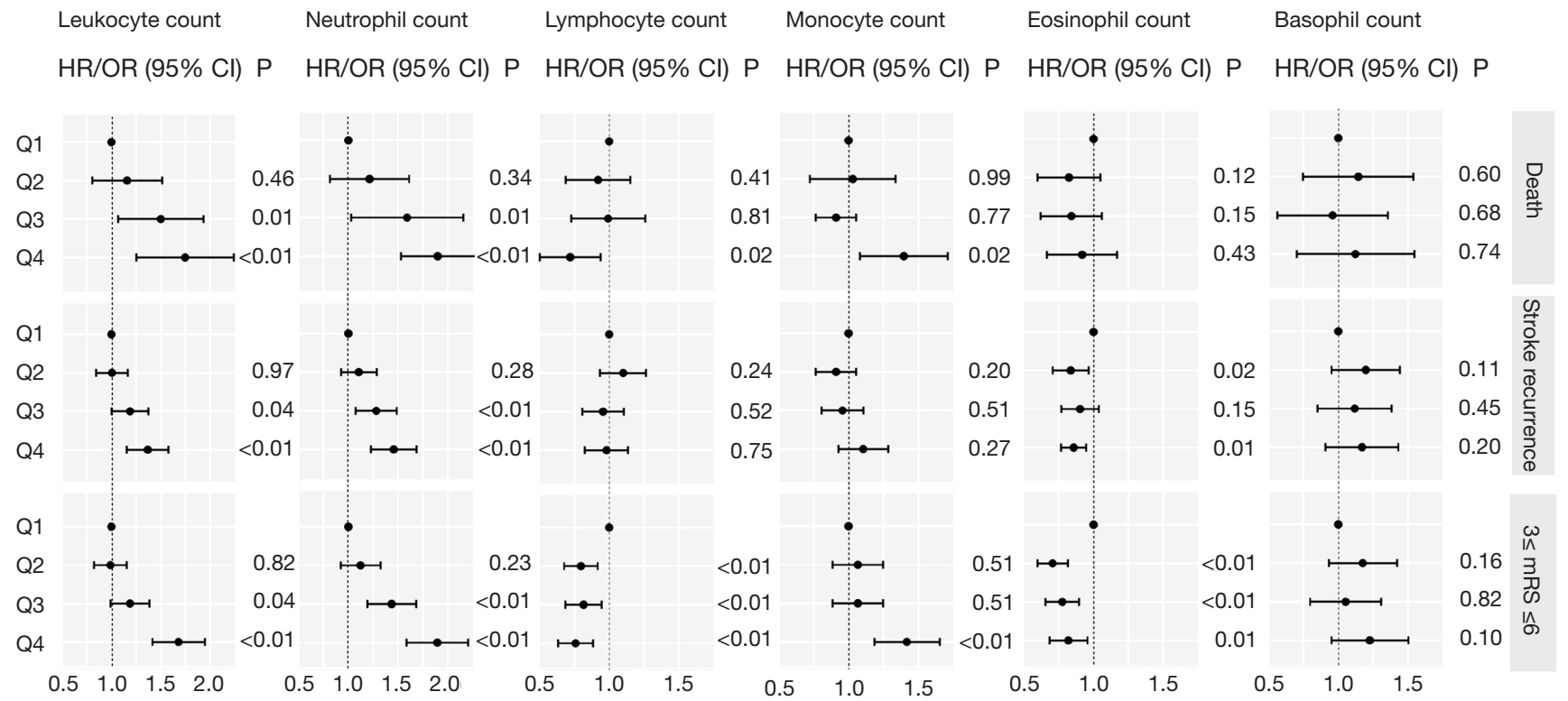

Figure 1 Adjusted HRs or ORs for adverse clinical outcomes at 1-year follow-up by leukocyte subtype counts. mRS, modified Rankin Scale; HR, hazard ratio; OR, odds ratio; CI, confidence interval.

percentage. There was also an obvious association between higher monocyte percentage and a reduced risk of stroke recurrence at 1-year follow-up. Moreover, higher basophil percentage was observed to be correlated with lower risks of death and poor functional outcomes at 1-year followup. After adjustments, the above relationships remained significant.

As shown in Table S5, the above associations between each leukocyte subtype percentage and the risks of adverse clinical outcomes were still valid at 3-month follow-up. Furthermore, higher monocyte percentage was related to a reduced risk of death at 3-month follow-up regardless of adjustments. Higher basophil percentage only had a significant association with stroke recurrence at 3-month follow-up before adjustments.

\section{Incremental predictive value of leukocyte subtypes}

We evaluated whether each leukocyte subtype could further increase the predictive value of conventional risk factors for adverse clinical outcomes at 1-year follow-up, shown in Table 2 and Table S6.

At 1-year follow-up, the $\mathrm{C}$ statistic of the conventional model for death significantly improved with the addition of neutrophil count, monocyte count, or lymphocyte percentage. The discriminatory power and risk reclassification also appeared to be slightly better with the addition of leukocyte count, neutrophil count, lymphocyte count, monocyte count, neutrophil percentage, lymphocyte percentage, or eosinophil percentage. As for stroke recurrence at 1-year follow-up, the addition of leukocyte count, neutrophil count, neutrophil percentage, lymphocyte percentage, or eosinophil percentage could improve the $\mathrm{C}$ statistic of the conventional model. Furthermore, adding leukocyte count, neutrophil count, neutrophil percentage, lymphocyte percentage, or monocyte percentage could make both discriminatory power and risk reclassification better in a bit. Meanwhile, eosinophil count or eosinophil percentage might only improve the risk reclassification. However, basophil percentage might even worsen the risk reclassification. There were also improvements in the $\mathrm{C}$ statistic for the conventional model for poor functional outcomes at 1-year follow-up with leukocyte count, neutrophil count, monocyte count, neutrophil percentage, or lymphocyte percentage. The discriminatory power and risk reclassification might be ameliorated by adding leukocyte count, neutrophil count, lymphocyte count, monocyte count, neutrophil percentage, or lymphocyte percentage. Similarly, eosinophil count or eosinophil percentage seemed only to improve the risk reclassification. 

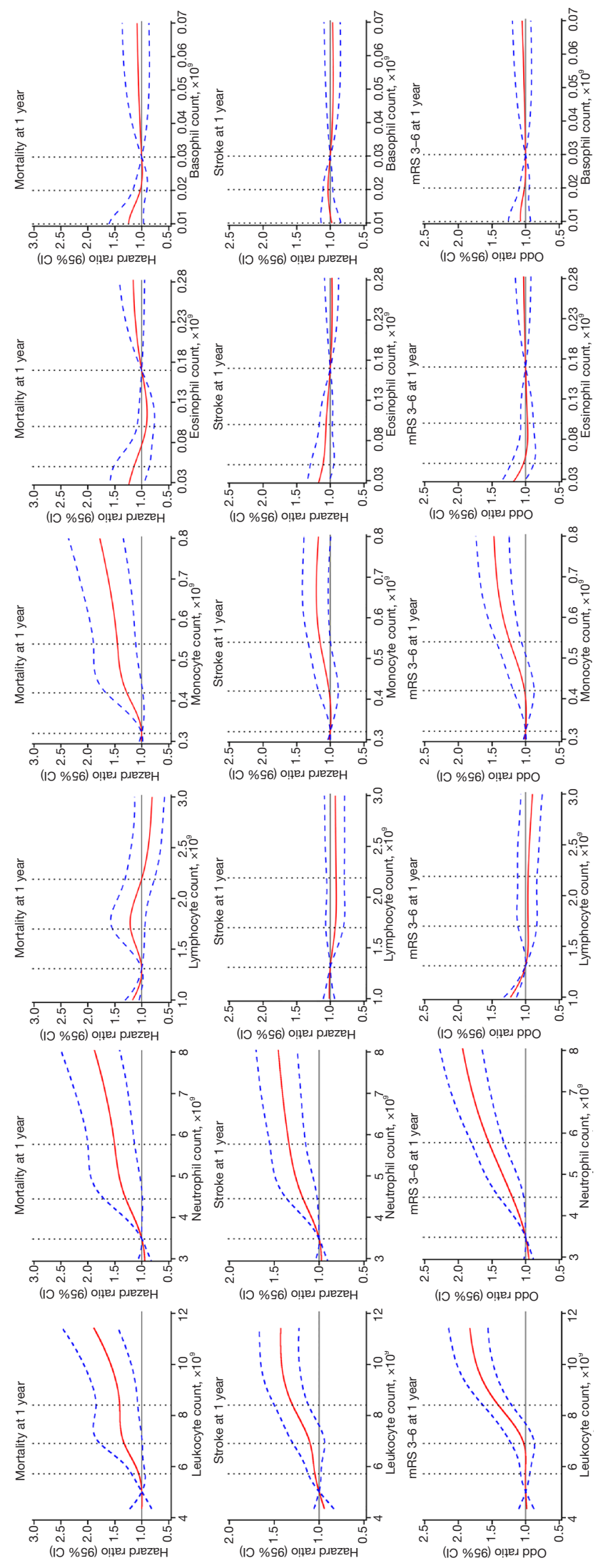

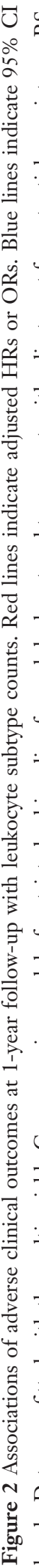




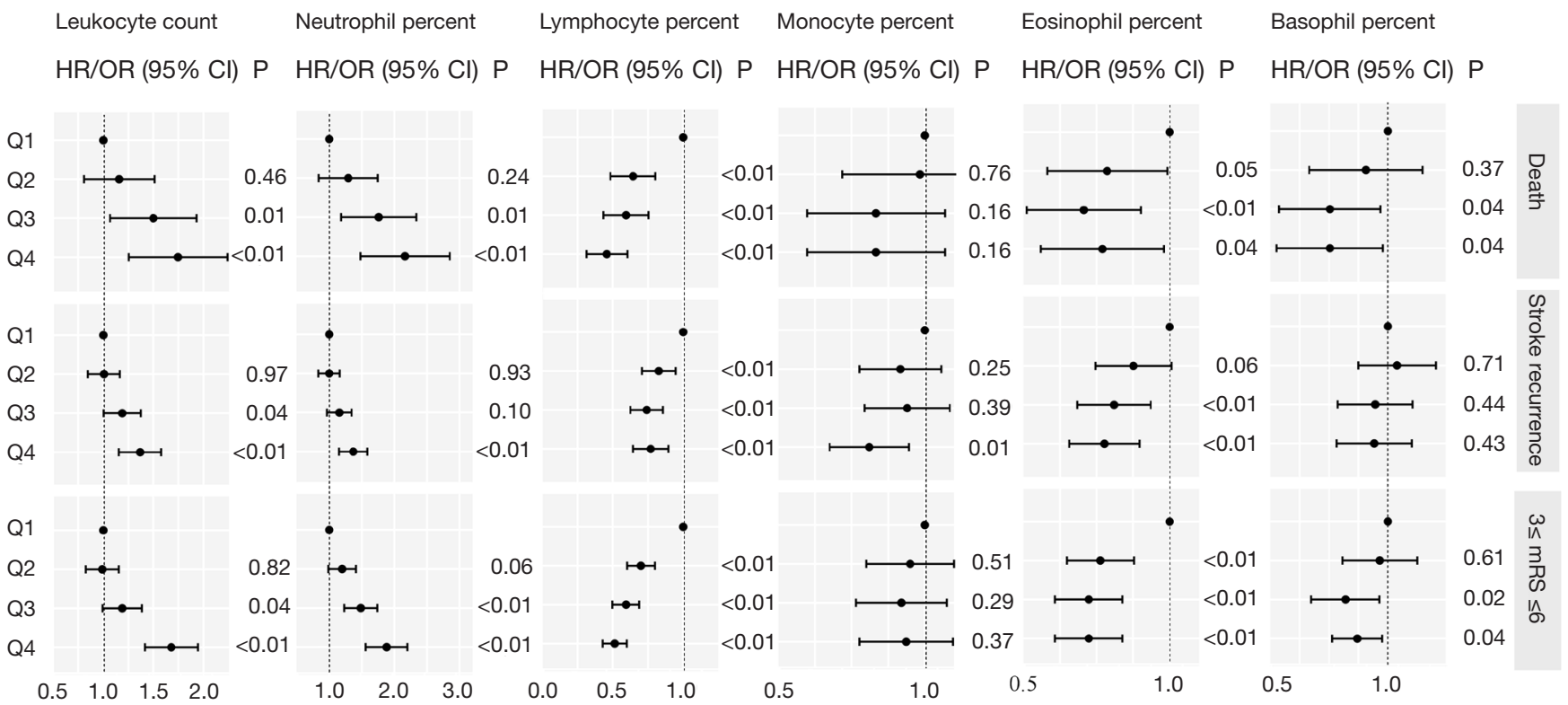

Figure 3 Adjusted HRs or ORs for adverse clinical outcomes at 1-year follow-up by leukocyte subtype percentages. mRS, modified Rankin Scale; HR, hazard ratio; OR, odds ratio; CI, confidence interval.

\section{Discussion}

This analysis demonstrated that different leukocyte subtypes had different relationships with adverse clinical outcomes at 3-month and 1-year follow-up in patients with acute ischemic cerebrovascular events. Higher counts of leukocytes, neutrophils, and monocytes were associated with elevated risks of adverse clinical outcomes. In contrast, higher counts of lymphocytes and eosinophils were related to reduced risks of adverse clinical outcomes. Meanwhile, basophil count seemed to not correlate with adverse clinical outcomes. Besides the counts of leukocyte subtypes, there were also significant associations between the percentages of leukocyte subtypes and adverse clinical outcomes.

Neutrophils are one of the most important leukocyte subtypes and are the first leukocyte subtype to upregulate expression substantially and invade injured brain tissue after acute ischemic stroke (14). It has been found in experimental models that the invasion of neutrophils could be detected as early as 5 hours after ischemic stroke onset and peak at 24 48 hours (15). Previous studies have shown that neutrophils could aggravate ischemic brain injury, and higher neutrophil counts are correlated with increased stroke severity, infarct volume, and worse functional outcomes $(12,16,17)$. Some experimental studies have suggested that depleting circulating neutrophils, inhibiting neutrophil infiltration, or blocking the neutrophil pro-inflammatory function could reduce infarct volume and improve neurological outcomes $(18,19)$. Furthermore, Semerano et al. reported that even after adjusting for the occurrence of infections, the association of higher neutrophil counts with worse 3 -month functional outcomes was still significant (3). Beyond neutrophil counts, Zhu et al.'s study revealed that high neutrophil ratio levels were also related to increased risks of new stroke and composite events in patients with minor ischemic stroke or TIA (20). Consistent with these prior studies, we found that patients with acute ischemic cerebrovascular events with higher neutrophil counts or percentages were more likely to suffer death, stroke recurrence, and poor functional outcomes at 3-month and 1-year follow-up. Nowadays, many mechanisms for neutrophil aggravation of ischemic brain injury have been proposed, for example, blocking microvessels, interacting with platelets, and releasing deleterious substances or inflammatory mediators $(14,19,21)$.

In contrast with neutrophils, it has been found that there is an exponential decrease in the peripheral circulating lymphocyte count in the hours immediately after ischemic stroke (4). Lower lymphocyte count has been revealed to be a marker of severe brain damage and is predictive of poor neurological improvement during the first week, poor functional outcomes at 3 months, and stroke-associated 







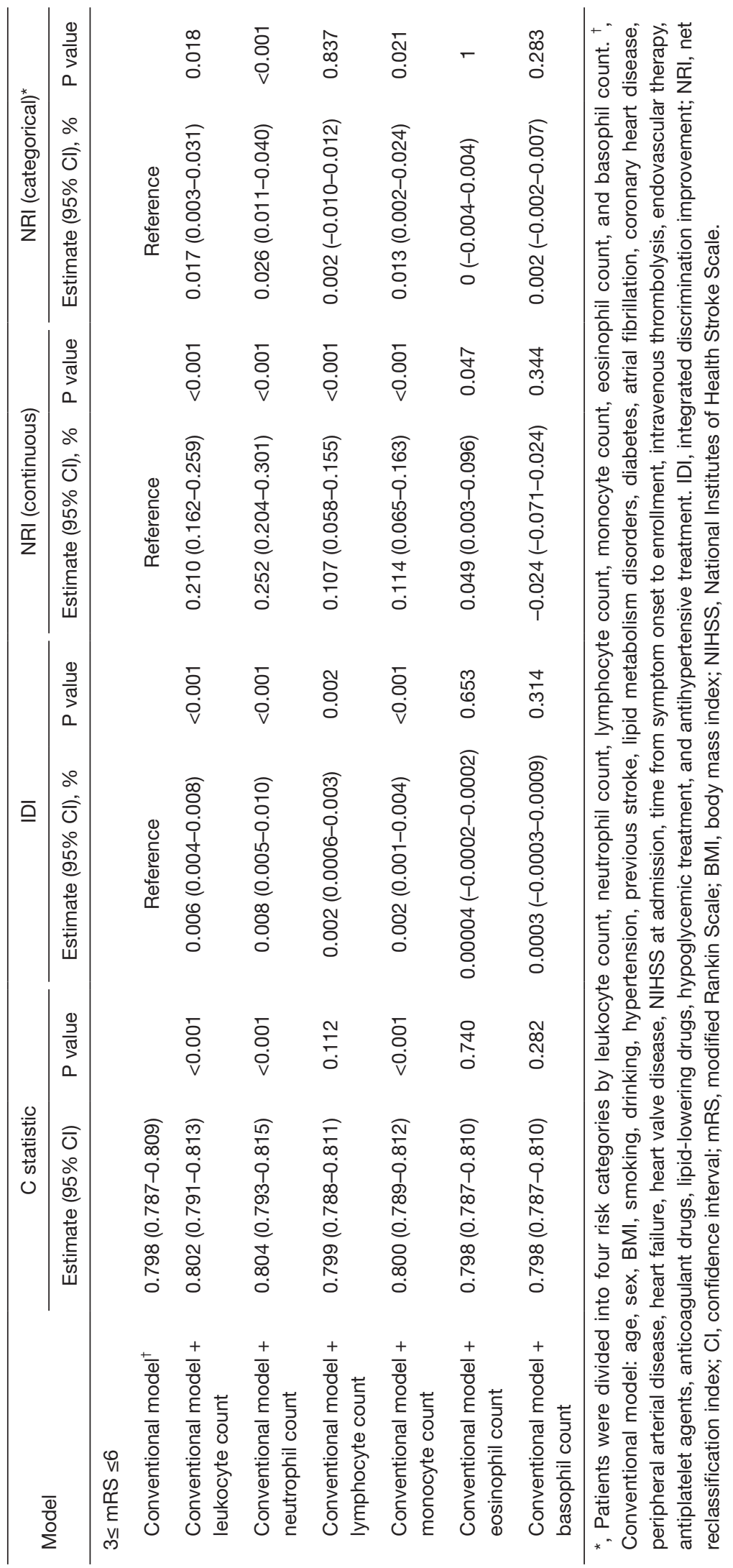


infections $(12,22)$. Our analyses demonstrated similar results in that a higher lymphocyte count was correlated with reduced risks of poor functional outcomes at 3-month and 1-year follow-up in patients with acute ischemic cerebrovascular events. Moreover, higher lymphocyte count might also be related to lower death and stroke recurrence risks at 3-month and 1-year follow-up. As for lymphocyte percentage, patients with higher lymphocyte percentages tended to have reduced incidences of death, stroke recurrence, and poor functional outcomes at 3-month and 1-year follow-up regardless of adjustments. In short, the above findings suggested that lymphocytes might play a protective role in ischemic brain areas; however, the underlying mechanisms are still not clear. One possible mechanism is that the percentages of the disease-limiting protective lymphocyte subtypes which can produce antiinflammatory cytokines and maintain immune homeostasis, such as regulatory $\mathrm{T}$ cells, increase in the brain after ischemic stroke $(23,24)$. Another possible mechanism is that lymphopenia may reflect increased sympathetic tone and serum cortisol levels, which are associated with increased production of proinflammatory cytokines $(12,25,26)$.

Although monocyte infiltration within ischemic brain tissue peaks at 7 days after acute ischemic stroke onset, peripheral circulating monocyte count increases earlier $(27,28)$. Meanwhile, it has been found that the temporal trends of different monocyte subtypes after stroke might not all be increased. For instance, the proportion of CD $14{ }^{\text {high }} \mathrm{CD} 16$ - monocytes do not significantly change, CD14 ${ }^{\mathrm{dim}} \mathrm{CD} 16+$ monocytes decrease, and only $\mathrm{CD} 14^{\text {high }} \mathrm{CD} 16+$ monocytes increase (29). Besides that, the effects of different monocyte subtypes on prognosis after stroke also vary, as Urra et al. suggested that classic $\mathrm{CD} 14^{\text {high }} \mathrm{CD} 16$ - monocytes had deleterious effects, whereas rare $\mathrm{CD} 14^{\mathrm{dim}} \mathrm{CD} 16+$ monocytes and $\mathrm{CD} 14^{\text {high }} \mathrm{CD} 16+$ monocytes were associated with clinical benefits (29). Overall, more studies have shown that monocyte count is positively correlated with the severity of brain injury and infarct volume after acute ischemic stroke and could be an independent predictor of poor clinical outcomes in patients $(4,28,30,31)$. Similarly, our results also demonstrated that higher monocyte count had an obvious association with elevated risks of death and poor functional outcomes at 3-month and 1-year follow-up in patients with acute ischemic cerebrovascular events; however, there was no relationship between monocyte count and stroke recurrence. In contrast, monocyte percentage had a negative association with death at 3-month follow-up and stroke recurrence at 3-month and 1-year follow-up, which might be due to the influence of leukocyte count as the denominator. The mechanism underlying the role of monocytes after ischemic stroke is complex. On the one hand, monocytes could contribute to the development of inflammation and promote thrombosis and vascular occlusion by producing inflammatory mediators and forming platelet-monocyte aggregates, which aggravate cerebral ischemic injury $(27,30)$. On the other hand, macrophages transformed by monocytes in the ischemic area might lead to angiogenesis stimulation, which could be considered beneficial (27).

Hypereosinophilia has been reported as an unusual cause of ischemic stroke due to thromboembolism from endomyocardial fibrosis or vascular endothelial toxicity of eosinophilic cells $(32,33)$. However, in patients without previous hypereosinophilia, eosinophils might play a protective role in the same way as lymphocytes after acute ischemic stroke. Previous studies have demonstrated that eosinophil count is negatively associated with stroke severity, risk of mortality, and poor functional outcomes in acute ischemic stroke patients $(3,34)$. It has even been shown that acute ischemic stroke patients with lower eosinophil counts are more likely to have functional impairment in limbs and difficulty in recovering (35). Moreover, higher eosinophil count at admission is suggested to be an independent predictive factor for lower odds of developing hemorrhagic transformation after treatment with recombinant tissue plasminogen activator for acute ischemic stroke (32). A similar relationship has been found between eosinophil percentage and acute ischemic stroke prognosis (34). Our results supported these previous studies in that higher eosinophil count was related to reduced risks of stroke recurrence at 1-year follow-up and poor functional outcomes at 3-month and 1-year follow-up in patients with acute ischemic cerebrovascular events. There also might be negative associations of eosinophil count with stroke recurrence at 3-month follow-up and death at 3-month and 1-year follow-up. Furthermore, lower risks of death, stroke recurrence, and poor functional outcomes at 3-month follow-up and 1-year follow-up were shown in patients with higher eosinophil percentages. There are a few hypotheses which may explain how eosinopenia leads to poor prognosis after acute ischemic stroke. Eosinophils can secrete numerous cytokines, growth factors, and chemokines to induce the activation of M2 phenotype microglia, which have neuroprotective properties and may facilitate the resolution of inflammation (32). Another mechanism may be the correlation with angiogenesis promotion, since 
eosinophils can produce vascular endothelial growth factor $(32,35,36)$.

Up to now, few studies have investigated the response of basophils after acute ischemic stroke, and data on the relationship between basophils and prognosis are also limited. Our analyses showed that there were no significant correlations between basophil count and death, stroke recurrence, or poor functional outcomes at 1-year followup in patients with acute ischemic cerebrovascular events; however, at 3-month follow-up, higher basophil count might only be associated with a reduced risk of poor functional outcomes. Basophil percentage was observed to be negatively related to death, and poor functional outcomes at 3-month and 1-year follow-up, and might also have a negative relationship with stroke recurrence at 3-month follow-up. However, we thought that this negative association between basophil percentage and the prognosis of acute ischemic cerebrovascular events was because of the leukocyte count's influence as the denominator. Based on these results, we speculated that basophils might play a limited role in developing acute ischemic cerebrovascular events due to their low content. Nevertheless, further studies are required.

Compared with the conventional model, we observed that the counts or percentages of leukocytes or each subtype, except for basophils, could slightly increase the predictive value of prognosis at 1-year follow-up in patients with acute ischemic cerebrovascular events. Of the leukocyte subtypes, neutrophils and lymphocytes seemed to have better predictive value. Therefore, as valuable peripheral blood biomarkers, leukocyte subtypes require further study to unravel their underlying mechanisms after acute ischemic cerebrovascular events and the therapeutic implications that might be derived.

There are several limitations to our study. Firstly, there is inevitable equipment heterogeneity across participating hospitals, which could have contributed to biased estimates. However, this might have had little impact because of daily practices and strict quality control. Secondly, our study only collected the counts and percentages of each leukocyte subtype within the first 24 hours after admission and did not evaluate the dynamic changes. Nevertheless, it might still provide valuable information on leukocyte subtypes' underlying mechanisms after acute ischemic cerebrovascular events. Thirdly, our study did not further assess whether there was a difference in each leukocyte subtype's relationship with adverse clinical outcomes between ischemic stroke patients and TIA patients. Finally, residual bias still exists due to the influence of comorbidities or environmental factors such as tumors, trauma, and acute toxicosis.

\section{Conclusions}

Different leukocyte subtypes had different relationships with adverse clinical outcomes at 3-month and 1-year follow-up in patients with acute ischemic cerebrovascular events. Additionally, leukocyte subtypes, except for basophils, could slightly increase the prognosis's predictive value at 1-year follow-up in patients with acute ischemic cerebrovascular events.

\section{Acknowledgments}

We thank all study participants, their relatives, the members of the survey teams at participating hospitals of the Third China National Stroke Registry, and the project development and management teams at the Beijing Tiantan Hospital.

Funding: This study was supported by grants from the National Key R\&D Program of China (2018YFC1312903), Beijing Municipal Science \& Technology Commission (D171100003017002, Z181100001818001), National Science and Technology Major Project (2017ZX09304018), Beijing Municipal Administration of Hospitals Incubating Program (PX2020021), and Beijing Excellent Talents Training Program (2018000021469G234).

\section{Footnote}

Reporting Checklist: The authors have completed the STROBE reporting checklist. Available at http://dx.doi. org/10.21037/atm-20-7931

Conflicts of Interest: All authors have completed the ICMJE uniform disclosure form (available at http://dx.doi. org/10.21037/atm-20-7931). The authors have no conflicts of interest to declare.

Ethical Statement: The authors are accountable for all aspects of the work in ensuring that questions related to the accuracy or integrity of any part of the work are appropriately investigated and resolved. Our study derived data from the CNSR-III. The ethics committee at Beijing Tiantan Hospital (IRB approval number: KY2015-001-01) and all study centers gave ethical approval of the CNSR- 
III study protocol. This study was conducted in accordance with the Declaration of Helsinki (as revised in 2013). All patients or their legal representatives provided written informed consent before being entered into the CNSR-III study.

Open Access Statement: This is an Open Access article distributed in accordance with the Creative Commons Attribution-NonCommercial-NoDerivs 4.0 International License (CC BY-NC-ND 4.0), which permits the noncommercial replication and distribution of the article with the strict proviso that no changes or edits are made and the original work is properly cited (including links to both the formal publication through the relevant DOI and the license). See: https://creativecommons.org/licenses/by-nc-nd/4.0/.

\section{References}

1. O'Connell GC, Chang JHC. Analysis of early strokeinduced changes in circulating leukocyte counts using transcriptomic deconvolution. Transl Neurosci 2018;9:161-6.

2. Iadecola C, Anrather J. The immunology of stroke: from mechanisms to translation. Nat Med 2011;17:796-808.

3. Semerano A, Strambo D, Martino G, et al. Leukocyte counts and ratios are predictive of stroke outcome and hemorrhagic complications independently of infections. Front Neurol 2020;11:201.

4. Gill D, Sivakumaran P, Aravind A, et al. Temporal trends in the levels of peripherally circulating leukocyte subtypes in the hours after ischemic stroke. J Stroke Cerebrovasc Dis 2018;27:198-202.

5. Zierath D, Tanzi P, Shibata D, et al. Cortisol is more important than metanephrines in driving changes in leukocyte counts after stroke. J Stroke Cerebrovasc Dis 2018;27:555-62.

6. Zheng X, Zeng N, Wang A, et al. Prognostic value of white blood cell in acute ischemic stroke patients. Curr Neurovasc Res 2018;15:151-7.

7. Nardi K, Milia P, Eusebi P, et al. Admission leukocytosis in acute cerebral ischemia: influence on early outcome. J Stroke Cerebrovasc Dis 2012;21:819-24.

8. Kotfis K, Bott-Olejnik M, Szylińska A, et al. Characteristics, risk factors and outcome of early-onset delirium in elderly patients with first ever acute ischemic stroke - a prospective observational cohort study. Clin Interv Aging 2019;14:1771-82.

9. Kotfis K, Bott-Olejnik M, Szylińska A, et al. Could neutrophil-to-lymphocyte ratio (NLR) serve as a potential marker for delirium prediction in patients with acute ischemic Stroke? A prospective observational study. J Clin Med 2019;8:1075.

10. Quan K, Wang A, Zhang X, et al. Leukocyte count and adverse clinical outcomes in acute ischemic stroke patients. Front Neurol 2019;10:1240.

11. Macrez R, Ali C, Toutirais O, et al. Stroke and the immune system: from pathophysiology to new therapeutic strategies. Lancet Neurol 2011;10:471-80.

12. Kim J, Song TJ, Park JH, et al. Different prognostic value of white blood cell subtypes in patients with acute cerebral infarction. Atherosclerosis 2012;222:464-7.

13. Wang Y, Jing J, Meng X, et al. The Third China National Stroke Registry (CNSR-III) for patients with acute ischaemic stroke or transient ischaemic attack: design, rationale and baseline patient characteristics. Stroke Vasc Neurol 2019;4:158-64.

14. Jickling GC, Liu D, Ander BP, et al. Targeting neutrophils in ischemic stroke: translational insights from experimental studies. J Cereb Blood Flow Metab 2015;35:888-901.

15. Jin R, Yang G, Li G. Inflammatory mechanisms in ischemic stroke: role of inflammatory cells. J Leukoc Biol 2010;87:779-89.

16. Tang $\mathrm{Y}, \mathrm{Xu} \mathrm{H}, \mathrm{Du} \mathrm{XL}$, et al. Gene expression in blood changes rapidly in neutrophils and monocytes after ischemic stroke in humans: a microarray study. J Cereb Blood Flow Metab 2006;26:1089-102.

17. Buck BH, Liebeskind DS, Saver JL, et al. Early neutrophilia is associated with volume of ischemic tissue in acute stroke. Stroke 2008;39:355-60.

18. McColl BW, Rothwell NJ, Allan SM. Systemic inflammatory stimulus potentiates the acute phase and CXC chemokine responses to experimental stroke and exacerbates brain damage via interleukin-1and neutrophil-dependent mechanisms. J Neurosci 2007;27:4403-12.

19. Ruhnau J, Schulze J, Dressel A, et al. Thrombosis, neuroinflammation, and poststroke infection: the multifaceted role of neutrophils in stroke. J Immunol Res 2017;2017:5140679.

20. Zhu B, Pan Y, Jing J, et al. Neutrophil counts, neutrophil ratio, and new stroke in minor ischemic stroke or TIA. Neurology 2018;90:e1870-8.

21. Kleinig TJ, Vink R. Suppression of inflammation in ischemic and hemorrhagic stroke: therapeutic options. Curr Opin Neurol 2009;22:294-301.

22. Urra X, Cervera Á, Villamor N, et al. Harms and benefits 
of lymphocyte subpopulations in patients with acute stroke. Neuroscience 2009;158:1174-83.

23. Liesz A, Hu X, Kleinschnitz C, et al. Functional Role of Regulatory Lymphocytes in Stroke: Facts and Controversies. Stroke 2015;46:1422-30.

24. Bonaventura A, Liberale L, Vecchié A, et al. Update on inflammatory biomarkers and treatments in ischemic stroke. Int J Mol Sci 2016;17:1967.

25. Park BJ, Shim JY, Lee HR, et al. Relationship of neutrophil-lymphocyte ratio with arterial stiffness and coronary calcium score. Clin Chim Acta 2011;412:925-9.

26. Acanfora D, Gheorghiade M, Trojano L, et al. Relative lymphocyte count: a prognostic indicator of mortality in elderly patients with congestive heart failure. Am Heart J 2001;142:167-73.

27. Bonifačić D, Toplak A, Benjak I, et al. Monocytes and monocyte chemoattractant protein 1 (MCP-1) as early predictors of disease outcome in patients with cerebral ischemic stroke. Wien Klin Wochenschr 2016;128:20-7.

28. Liberale L, Montecucco F, Bonaventura A, et al. Monocyte count at onset predicts poststroke outcomes during a 90day follow-up. Eur J Clin Invest 2017;47:702-10.

29. Urra X, Villamor N, Amaro S, et al. Monocyte subtypes predict clinical course and prognosis in human stroke. J Cereb Blood Flow Metab 2009;29:994-1002.

Cite this article as: Wang A, Quan K, Tian X, Zuo Y, Meng X, Chen P, Li H, Wang Y. Leukocyte subtypes and adverse clinical outcomes in patients with acute ischemic cerebrovascular events. Ann Transl Med 2021;9(9):748. doi: 10.21037/atm-207931
30. Dong X, Nao J, Gao Y. Peripheral monocyte count predicts outcomes in patients with acute ischemic stroke treated with rtPA thrombolysis. Neurotox Res 2020;37:469-77.

31. Bolayir A, Gokce SF, Cigdem B, et al. Monocyte/highdensity lipoprotein ratio predicts the mortality in ischemic stroke patients. Neurol Neurochir Pol 2018;52:150-5.

32. Jucevičiūtė N, Mikužis P, Balnytė R. Absolute blood eosinophil count could be a potential biomarker for predicting haemorrhagic transformation after intravenous thrombolysis for acute ischaemic stroke. BMC Neurol 2019;19:127.

33. Khwaja GA, Duggal A, Kulkarni A, et al. Hypereosinophilia-an unusual cause of multiple embolic strokes and multi-organ dysfunction. J Clin Diagn Res 2013;7:2316-8.

34. Wang J, Ma L, Lin T, et al. The significance of eosinophils in predicting the severity of acute ischemic stroke. Oncotarget 2017;8:104238-46.

35. Guo LB, Liu S, Zhang F, et al. The role of eosinophils in stroke: a pilot study. Eur Rev Med Pharmacol Sci 2015;19:3643-8.

36. Puxeddu I, Alian A, Piliponsky AM, et al. Human peripheral blood eosinophils induce angiogenesis. Int J Biochem Cell Biol 2005;37:628-36. 
Patients recruited in the Third China National Stroke Registry (CNSR-III) $\mathrm{N}=15,166$

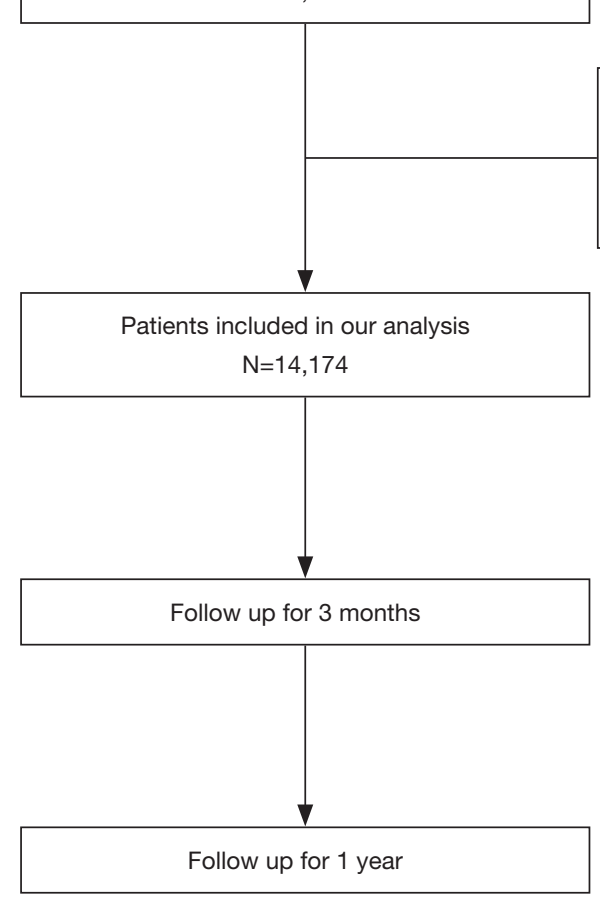

Exclusion criteria:

Patients without available complete blood count

on admission or lost to follow-up

$\mathrm{N}=992$

Figure S1 Flow diagram. 
Table S1 Baseline characteristics of included patients and excluded patients

\begin{tabular}{|c|c|c|c|}
\hline Baseline characteristics & Excluded patients $(n=992)$ & Included patients $(n=14,174)$ & $\mathrm{P}$ \\
\hline Age, median [IQR], y & 64 [55-72] & $62[54-70]$ & 0.0005 \\
\hline Female, n (\%) & $309(31.15)$ & $4,493(31.70)$ & 0.719 \\
\hline $\mathrm{BMI}, \mathrm{kg} / \mathrm{m}^{2}$, median $[\mathrm{IQR}]$ & 24.36 [22.58-26.36] & 24.49 [22.6-26.57] & 0.4984 \\
\hline Current drinking, $\mathrm{n}(\%)$ & $165(16.63)$ & $2,300(16.23)$ & 0.7375 \\
\hline \multicolumn{4}{|l|}{ Medical history, n (\%) } \\
\hline Previous stroke & $207(20.87)$ & $3,148(22.21)$ & 0.3246 \\
\hline Previous TIA & $22(2.22)$ & $394(2.78)$ & 0.2948 \\
\hline Atrial fibrillation & $76(7.66)$ & $943(6.65)$ & 0.2201 \\
\hline Heart failure & $8(0.81)$ & $86(0.61)$ & 0.4385 \\
\hline Heart valve disease & $6(0.60)$ & $54(0.38)$ & 0.2776 \\
\hline Hypertension & $651(65.63)$ & $8,843(62.39)$ & 0.0417 \\
\hline Lipid metabolism disorder & $55(5.54)$ & $1,136(8.01)$ & 0.0052 \\
\hline Peripheral arterial disease & $8(0.81)$ & $110(0.78)$ & 0.9161 \\
\hline Infection within 2 weeks before admission & $25(2.52)$ & $425(3.00)$ & 0.3907 \\
\hline NIHSS score at admission, median [IQR] & $3[2-7]$ & $3[1-6]$ & $<0.0001$ \\
\hline \multicolumn{4}{|l|}{ Therapy, n (\%) } \\
\hline
\end{tabular}

IQR, interquartile range; BMI, body mass index; TIA, transient ischemic attack; NIHSS, National Institutes of Health Stroke Scale. 
Table S2 HRs or ORs and 95\% CIs of adverse clinical outcomes at 1-year follow-up by leukocyte count, neutrophil count, lymphocyte count, monocyte count, eosinophil count, basophil count

\begin{tabular}{|c|c|c|c|c|c|c|c|c|c|c|c|c|c|c|c|}
\hline \multirow{2}{*}{ Variable } & \multicolumn{5}{|c|}{ Death } & \multicolumn{5}{|c|}{ Stroke recurrence } & \multicolumn{5}{|c|}{$3 \leq \mathrm{mRS} \leq 6$} \\
\hline & Q1 & Q2 & Q3 & Q4 & $\mathrm{P}$ & Q1 & Q2 & Q3 & Q4 & $\mathrm{P}$ & Q1 & Q2 & Q3 & Q4 & $P$ \\
\hline Leukocyte count, n (\%) & $82(2.31)$ & $87(2.46)$ & $116(3.27)$ & $161(4.54)$ & & $298(8.39)$ & $297(8.41)$ & $350(9.88)$ & 416 (11.72) & & $376(10.59)$ & 366 (10.37) & 451 (12.73) & $659(18.57)$ & \\
\hline Crude HR/OR (95\% Cl) & Reference & $1.06(0.78-1.44)$ & $1.44(1.09-1.92)$ & $1.90(1.45-2.49)$ & $<0.0001$ & Reference & $0.98(0.83-1.15)$ & $1.17(1.00-1.37)$ & $1.37(1.18-1.59)$ & $<0.0001$ & Reference & $0.95(0.82-1.12)$ & $1.22(1.05-1.41)$ & $1.83(1.59-2.11)$ & $<0.0001$ \\
\hline Adjusted HR/OR (95\% Cl) & Reference & $1.12(0.83-1.53)$ & $1.46(1.09-1.95)$ & $1.70(1.28-2.26)$ & $<0.0001$ & Reference & $1.00(0.85-1.17)$ & $1.18(1.01-1.38)$ & $1.36(1.16-1.58)$ & $<0.0001$ & Reference & $0.98(0.83-1.16)$ & $1.18(1.00-1.39)$ & $1.67(1.42-1.95)$ & $<0.0001$ \\
\hline Neutrophil count, $\mathrm{n}(\%)$ & $69(1.95)$ & $80(2.26)$ & $111(3.13)$ & $186(5.25)$ & & $274(7.72)$ & $301(8.51)$ & $363(10.25)$ & $423(11.93)$ & & $308(8.68)$ & $356(10.06)$ & 486 (13.72) & $702(19.80)$ & \\
\hline Crude HR/OR (95\% Cl) & Reference & $1.16(0.84-1.60)$ & $1.60(1.18-2.17)$ & $2.58(1.95-3.41)$ & $<0.0001$ & Reference & $1.09(0.93-1.29)$ & $1.29(1.10-1.51)$ & $1.51(1.30-1.77)$ & $<0.0001$ & Reference & $1.17(1.00-1.38)$ & $1.60(1.37-1.87)$ & $2.47(2.13-2.86)$ & $<0.0001$ \\
\hline Adjusted HR/OR (95\% Cl) & Reference & $1.17(0.84-1.63)$ & $1.48(1.09-2.02)$ & $2.00(1.49-2.69)$ & $<0.0001$ & Reference & $1.10(0.93-1.29)$ & $1.27(1.08-1.49)$ & $1.45(1.23-1.69)$ & $<0.0001$ & Reference & $1.11(0.93-1.33)$ & $1.42(1.20-1.69)$ & $1.88(1.59-2.21)$ & $<0.0001$ \\
\hline Crude HR/OR (95\% Cl) & Reference & $0.63(0.50-0.80)$ & $0.57(0.44-0.74)$ & $0.38(0.28-0.50)$ & $<0.0001$ & Reference & $1.03(0.89-1.19)$ & $0.86(0.74-1.01)$ & $0.87(0.75-1.02)$ & 0.0181 & Reference & $0.63(0.55-0.71)$ & $0.54(0.47-0.63)$ & $0.46(0.40-0.53)$ & $<0.0001$ \\
\hline Adjusted HR/OR (95\% Cl) & Reference & $0.90(0.70-1.16)$ & $0.97(0.74-1.27)$ & $0.70(0.51-0.95)$ & 0.0564 & Reference & $1.09(0.94-1.27)$ & $0.95(0.81-1.11)$ & $0.97(0.83-1.14)$ & 0.412 & Reference & $0.79(0.68-0.92)$ & $0.81(0.69-0.95)$ & $0.75(0.64-0.89)$ & $<0.0001$ \\
\hline Monocyte count, $\mathrm{n}(\%)$ & $87(2.49)$ & $94(2.64)$ & $99(2.74)$ & $166(4.72)$ & & 347 (9.93) & $311(8.75)$ & $330(9.14)$ & $373(10.61)$ & & $377(10.79)$ & 408 (11.48) & $446(12.35)$ & $621(17.66)$ & \\
\hline Crude HR/OR (95\% Cl) & Reference & $1.04(0.77-1.40)$ & $1.08(0.80-1.46)$ & $1.77(1.34-2.33)$ & $<0.0001$ & Reference & $0.89(0.76-1.05)$ & $0.95(0.81-1.11)$ & $1.12(0.95-1.31)$ & 0.1165 & Reference & $1.03(0.88-1.20)$ & $1.05(0.90-1.23)$ & $1.50(1.29-1.74)$ & $<0.0001$ \\
\hline Adjusted HR/OR (95\% Cl) & Reference & $1.00(0.74-1.35)$ & $1.05(0.77-1.42)$ & $1.42(1.07-1.89)$ & 0.0086 & Reference & $0.90(0.77-1.06)$ & $0.95(0.81-1.11)$ & $1.10(0.93-1.29)$ & 0.2051 & Reference & $1.06(0.89-1.25)$ & $1.06(0.89-1.25)$ & $1.41(1.19-1.66)$ & $<0.0001$ \\
\hline Eosinophil count, $\mathrm{n}(\%)$ & $155(4.75)$ & $92(2.61)$ & $105(2.64)$ & $94(2.76)$ & & $375(11.49)$ & $314(8.91)$ & $379(9.53)$ & $293(8.60)$ & & $608(18.63)$ & $390(11.07)$ & $453(11.39)$ & $401(11.77)$ & \\
\hline Crude HR/OR (95\% Cl) & Reference & $0.58(0.44-0.75)$ & $0.58(0.45-0.75)$ & $0.61(0.47-0.79)$ & $<0.0001$ & Reference & $0.80(0.68-0.93)$ & $0.84(0.73-0.97)$ & $0.76(0.65-0.89)$ & 0.0021 & Reference & $0.55(0.48-0.63)$ & $0.56(0.49-0.64)$ & $0.58(0.50-0.67)$ & $<0.0001$ \\
\hline Basophil count, $\mathrm{n}(\%)$ & $60(3.15)$ & $228(3.39)$ & $62(2.71)$ & $96(2.94)$ & & $171(8.98)$ & $681(10.14)$ & $209(9.15)$ & $300(9.19)$ & & 255 (13.39) & $918(13.66)$ & $265(11.60)$ & 414 (12.68) & \\
\hline Crude HR/OR (95\% Cl) & Reference & $1.06(0.74-1.50)$ & $0.77(0.51-1.17)$ & $0.85(0.58-1.25)$ & 0.0762 & Reference & $1.21(0.98-1.48)$ & $1.08(0.85-1.38)$ & $1.13(0.91-1.41)$ & 0.9409 & Reference & $1.10(0.91-1.33)$ & $0.86(0.69-1.07)$ & $0.93(0.76-1.14)$ & 0.1003 \\
\hline Adjusted HR/OR (95\% Cl) & Reference & $1.10(0.77-1.56)$ & $0.91(0.59-1.38)$ & $1.07(0.73-1.57)$ & 0.8623 & Reference & $1.18(0.96-1.45)$ & $1.10(0.86-1.39)$ & $1.15(0.92-1.44)$ & 0.6591 & Reference & $1.16(0.94-1.43)$ & $1.03(0.81-1.32)$ & $1.21(0.96-1.51)$ & 0.5955 \\
\hline
\end{tabular}
mRS, modified Rankin Scale; HR, hazard ratio; OR, odds ratio; Cl, confidence interval. 
Table S3 HRs or ORs and 95\% CIs of adverse clinical outcomes at 3-month follow-up by leukocyte count, neutrophil count, lymphocyte count, monocyte count, eosinophil count, basophil count

\begin{tabular}{|c|c|c|c|c|c|c|c|c|c|c|c|c|c|c|c|}
\hline \multirow{2}{*}{ Variable } & \multicolumn{5}{|c|}{ Death } & \multicolumn{5}{|c|}{ Stroke recurrence } & \multicolumn{5}{|c|}{$3 \leq \mathrm{mRS} \leq 6$} \\
\hline & Q1 & Q2 & Q3 & Q4 & $\mathrm{P}$ & Q1 & Q2 & Q3 & Q4 & $\mathrm{P}$ & Q1 & Q2 & Q3 & Q4 & $\mathrm{P}$ \\
\hline Leukocyte count, n (\%) & $28(0.79)$ & $34(0.96)$ & $46(1.30)$ & $96(2.70)$ & & $187(5.26)$ & $180(5.10)$ & $215(6.07)$ & $290(8.17)$ & & $360(10.14)$ & $376(10.65)$ & $474(13.38)$ & $705(19.86)$ & \\
\hline Crude HR/OR (95\% Cl) & Reference & $1.20(0.73-1.99)$ & $1.68(1.05-2.70)$ & $3.17(2.07-4.87)$ & $<0.0001$ & Reference & $0.95(0.77-1.17)$ & $1.14(0.93-1.39)$ & $1.54(1.28-1.86)$ & $<0.0001$ & Reference & $1.03(0.88-1.20)$ & $1.36(1.17-1.59)$ & $2.15(1.86-2.48)$ & $<0.0001$ \\
\hline Adjusted HR/OR (95\% Cl) & Reference & $1.33(0.80-2.22)$ & $1.75(1.08-2.85)$ & $2.79(1.78-4.39)$ & $<0.0001$ & Reference & $0.97(0.79-1.19)$ & $1.13(0.93-1.39)$ & $1.48(1.22-1.80)$ & $<0.0001$ & Reference & $1.04(0.88-1.23)$ & $1.29(1.09-1.52)$ & $1.85(1.57-2.17)$ & $<0.0001$ \\
\hline Neutrophil count, n (\%) & $21(0.59)$ & $27(0.76)$ & $44(1.24)$ & $112(3.16)$ & & $164(4.62)$ & $188(5.31)$ & $220(6.21)$ & $300(8.46)$ & & $300(8.46)$ & $341(9.64)$ & $507(14.31)$ & $767(21.63)$ & \\
\hline Crude HR/OR (95\% Cl) & Reference & $1.32(0.74-2.34)$ & $2.04(1.21-3.45)$ & $4.91(3.06-7.87)$ & $<0.0001$ & Reference & $1.14(0.92-1.41)$ & $1.30(1.06-1.60)$ & $1.81(1.49-2.19)$ & $<0.0001$ & Reference & $1.15(0.97-1.35)$ & $1.74(1.49-2.04)$ & $2.90(2.50-3.37)$ & $<0.0001$ \\
\hline Adjusted HR/OR $(95 \% \mathrm{Cl})$ & Reference & $1.36(0.76-2.42)$ & $1.94(1.14-3.31)$ & $3.72(2.26-6.10)$ & $<0.0001$ & Reference & $1.14(0.92-1.40)$ & $1.27(1.04-1.56)$ & $1.67(1.37-2.04)$ & $<0.0001$ & Reference & $1.06(0.88-1.26)$ & $1.52(1.28-1.80)$ & $2.12(1.79-2.50)$ & $<0.0001$ \\
\hline Crude HR/OR (95\% Cl) & Reference & $0.63(0.44-0.89)$ & $0.52(0.36-0.77)$ & $0.36(0.23-0.56)$ & $<0.0001$ & Reference & $1.03(0.86-1.23)$ & $0.82(0.68-0.99)$ & $0.79(0.65-0.96)$ & 0.0027 & Reference & $0.63(0.55-0.71)$ & $0.55(0.48-0.63)$ & $0.43(0.37-0.49)$ & $<0.0001$ \\
\hline Adjusted HR/OR (95\% Cl) & Reference & $1.04(0.71-1.52)$ & $1.08(0.72-1.63)$ & $0.81(0.51-1.29)$ & 0.5481 & Reference & $1.09(0.91-1.30)$ & $0.90(0.74-1.09)$ & $0.87(0.71-1.07)$ & 0.0674 & Reference & $0.77(0.66-0.89)$ & $0.76(0.65-0.89)$ & $0.63(0.53-0.75)$ & $<0.0001$ \\
\hline Monocyte count, n (\%) & $36(1.03)$ & $41(1.15)$ & $46(1.27)$ & $81(2.30)$ & & $222(6.36)$ & $198(5.57)$ & $215(5.95)$ & $237(6.74)$ & & 407 (11.65) & 438 (12.32) & 445 (12.32) & $625(17.78)$ & \\
\hline Crude HR/OR (95\% Cl) & Reference & $1.05(0.67-1.66)$ & $1.15(0.73-1.81)$ & $1.91(1.25-2.90)$ & 0.001 & Reference & $0.90(0.74-1.09)$ & $0.98(0.80-1.19)$ & $1.12(0.92-1.36)$ & 0.1833 & Reference & $1.02(0.88-1.19)$ & $1.00(0.86-1.16)$ & $1.47(1.27-1.71)$ & $<0.0001$ \\
\hline Adjusted HR/OR (95\% Cl) & Reference & $0.95(0.59-1.51)$ & $1.22(0.77-1.93)$ & $1.44(0.93-2.24)$ & 0.043 & Reference & $0.91(0.75-1.11)$ & $0.99(0.81-1.20)$ & $1.10(0.90-1.34)$ & 0.2606 & Reference & $1.04(0.88-1.23)$ & $1.01(0.85-1.19)$ & $1.38(1.17-1.63)$ & $<0.0001$ \\
\hline Eosinophil count, n (\%) & $88(2.70)$ & $36(1.02)$ & $43(1.08)$ & $37(1.09)$ & & $249(7.63)$ & $198(5.62)$ & $236(5.93)$ & $189(5.55)$ & & $652(19.98)$ & 409 (11.61) & $467(11.74)$ & $387(11.36)$ & \\
\hline Crude HR/OR (95\% Cl) & Reference & $0.41(0.27-0.60)$ & $0.43(0.30-0.63)$ & $0.43(0.29-0.63)$ & $<0.0001$ & Reference & $0.74(0.61-0.89)$ & $0.79(0.65-0.94)$ & $0.72(0.60-0.88)$ & 0.0031 & Reference & $0.53(0.46-0.61)$ & $0.53(0.46-0.61)$ & $0.51(0.44-0.59)$ & $<0.0001$ \\
\hline Basophil count, $n$ (\%) & $21(1.10)$ & $109(1.62)$ & $33(1.44)$ & $41(1.26)$ & & $99(5.20)$ & $442(6.58)$ & $132(5.78)$ & $199(6.09)$ & & $279(14.65)$ & $947(14.09)$ & $275(12.04)$ & $414(12.68)$ & \\
\hline Crude HR/OR (95\% Cl) & Reference & $1.23(0.70-2.15)$ & $0.92(0.49-1.75)$ & $0.83(0.46-1.52)$ & 0.0775 & Reference & $1.22(0.94-1.58)$ & $1.06(0.78-1.44)$ & $1.16(0.88-1.54)$ & 0.9639 & Reference & $0.97(0.81-1.17)$ & $0.76(0.61-0.94)$ & $0.79(0.65-0.97)$ & 0.0063 \\
\hline Adjusted HR/OR (95\% Cl) & Reference & $1.22(0.69-2.16)$ & $1.12(0.59-2.14)$ & $0.98(0.53-1.83)$ & 0.4881 & Reference & $1.20(0.92-1.55)$ & $1.08(0.80-1.47)$ & $1.20(0.91-1.60)$ & 0.5488 & Reference & $1.01(0.82-1.24)$ & $0.90(0.70-1.14)$ & $0.99(0.79-1.23)$ & 0.5839 \\
\hline
\end{tabular}


Table S4 HRs or ORs and 95\% CIs of adverse clinical outcomes at 1-year follow-up by leukocyte count, neutrophil percent, lymphocyte percent, monocyte percent, eosinophil percent, basophil percent

\begin{tabular}{|c|c|c|c|c|c|c|c|c|c|c|c|c|c|c|c|}
\hline \multirow{2}{*}{ Variable } & \multicolumn{5}{|c|}{ Death } & \multicolumn{5}{|c|}{ Stroke recurrence } & \multicolumn{5}{|c|}{$3 \leq \mathrm{mRS} \leq 6$} \\
\hline & Q1 & Q2 & Q3 & Q4 & $P$ & Q1 & Q2 & Q3 & Q4 & $\mathrm{P}$ & Q1 & Q2 & Q3 & Q4 & $\mathrm{P}$ \\
\hline Leukocyte count, n (\%) & $82(2.31)$ & $87(2.46)$ & $116(3.27)$ & $161(4.54)$ & & 298 (8.39) & $297(8.41)$ & $350(9.88)$ & $416(11.72)$ & & $376(10.59)$ & $366(10.37)$ & 451 (12.73) & $659(18.57)$ & \\
\hline Crude HR/OR (95\% Cl) & Reference & $1.06(0.78-1.44)$ & $1.44(1.09-1.92)$ & $1.90(1.45-2.49)$ & $<0.0001$ & Reference & $0.98(0.83-1.15)$ & $1.17(1.00-1.37)$ & $1.37(1.18-1.59)$ & $<0.0001$ & Reference & $0.95(0.82-1.12)$ & $1.22(1.05-1.41)$ & $1.83(1.59-2.11)$ & $<0.0001$ \\
\hline Neutrophil percent, n (\%) & $54(1.52)$ & $76(2.14)$ & $113(3.19)$ & $203(5.73)$ & & $279(7.87)$ & $294(8.30)$ & $348(9.82)$ & 440 (12.42) & & $272(7.68)$ & $352(9.93)$ & $475(13.40)$ & $753(21.25)$ & \\
\hline Crude HR/OR (95\% Cl) & Reference & $1.32(0.93-1.88)$ & $2.02(1.46-2.80)$ & $3.59(2.64-4.87)$ & $<0.0001$ & Reference & $0.99(0.84-1.17)$ & $1.19(1.01-1.40)$ & $1.49(1.28-1.74)$ & $<0.0001$ & Reference & $1.28(1.08-1.51)$ & $1.82(1.55-2.14)$ & $3.10(2.66-3.61)$ & $<0.0001$ \\
\hline Adjusted HR/OR (95\% Cl) & Reference & $1.24(0.87-1.77)$ & $1.70(1.22-2.37)$ & $2.09(1.52-2.88)$ & $<0.0001$ & Reference & $0.99(0.84-1.17)$ & $1.15(0.97-1.35)$ & $1.36(1.16-1.59)$ & $<0.0001$ & Reference & $1.19(0.99-1.42)$ & $1.47(1.24-1.75)$ & $1.86(1.57-2.21)$ & $<0.0001$ \\
\hline Lymphocyte percent, $n(\%)$ & $222(6.27)$ & $96(2.71)$ & $76(2.14)$ & $52(1.47)$ & & $440(12.42)$ & $340(9.59)$ & $291(8.21)$ & $290(8.19)$ & & $800(22.58)$ & $450(12.70)$ & 341 (9.62) & $261(7.37)$ & \\
\hline Crude HR/OR (95\% Cl) & Reference & $0.43(0.34-0.55)$ & $0.36(0.27-0.46)$ & $0.25(0.18-0.34)$ & $<0.0001$ & Reference & $0.77(0.67-0.89)$ & $0.66(0.57-0.77)$ & $0.69(0.59-0.80)$ & $<0.0001$ & Reference & $0.52(0.46-0.60)$ & $0.39(0.33-0.44)$ & $0.30(0.26-0.36)$ & $<0.0001$ \\
\hline Adjusted HR/OR (95\% Cl) & Reference & $0.63(0.49-0.81)$ & $0.58(0.44-0.76)$ & $0.44(0.32-0.61)$ & $<0.0001$ & Reference & $0.82(0.71-0.95)$ & $0.73(0.63-0.86)$ & $0.76(0.65-0.90)$ & $<0.0001$ & Reference & $0.70(0.60-0.80)$ & $0.59(0.50-0.69)$ & $0.51(0.43-0.60)$ & $<0.0001$ \\
\hline Monocyte percent, $\mathrm{n}(\%)$ & $110(3.10)$ & $115(3.24)$ & $101(2.85)$ & $120(3.39)$ & & $379(10.70)$ & $343(9.68)$ & $339(9.57)$ & $300(8.47)$ & & $457(12.90)$ & $449(12.67)$ & $440(12.42)$ & $506(14.28)$ & \\
\hline Crude HR/OR (95\% Cl) & Reference & $0.98(0.75-1.28)$ & $0.84(0.63-1.11)$ & $0.93(0.70-1.22)$ & 0.4095 & Reference & $0.92(0.79-1.07)$ & $0.93(0.79-1.08)$ & $0.81(0.69-0.96)$ & 0.0215 & Reference & $0.90(0.78-1.04)$ & $0.85(0.73-0.99)$ & $0.91(0.79-1.06)$ & 0.1248 \\
\hline Adjusted HR/OR (95\% Cl) & Reference & $0.96(0.73-1.26)$ & $0.81(0.61-1.08)$ & $0.81(0.61-1.08)$ & 0.0919 & Reference & $0.91(0.78-1.06)$ & $0.93(0.80-1.09)$ & $0.80(0.68-0.95)$ & 0.0204 & Reference & $0.95(0.80-1.11)$ & $0.91(0.77-1.08)$ & $0.93(0.78-1.10)$ & 0.3154 \\
\hline Eosinophil percent, $\mathrm{n}(\%)$ & $177(5.00)$ & $93(2.62)$ & $84(2.38)$ & $92(2.59)$ & & $412(11.63)$ & $339(9.55)$ & $315(8.91)$ & $295(8.32)$ & & $671(18.94)$ & $414(11.66)$ & $386(10.92)$ & 381 (10.74) & \\
\hline Crude HR/OR (95\% Cl) & Reference & $0.54(0.42-0.70)$ & $0.50(0.38-0.65)$ & $0.54(0.42-0.70)$ & $<0.0001$ & Reference & $0.83(0.72-0.96)$ & $0.76(0.65-0.88)$ & $0.72(0.62-0.84)$ & $<0.0001$ & Reference & $0.57(0.50-0.66)$ & $0.53(0.46-0.61)$ & $0.52(0.45-0.60)$ & $<0.0001$ \\
\hline Adjusted HR/OR (95\% Cl) & Reference & $0.77(0.59-1.00)$ & $0.69(0.52-0.91)$ & $0.75(0.57-0.99)$ & 0.018 & Reference & $0.87(0.75-1.01)$ & $0.80(0.69-0.94)$ & $0.77(0.66-0.90)$ & $<0.0001$ & Reference & $0.76(0.65-0.88)$ & $0.72(0.61-0.84)$ & $0.72(0.61-0.84)$ & $<0.0001$ \\
\hline Crude HR/OR (95\% Cl) & Reference & $0.79(0.61-1.03)$ & $0.60(0.45-0.80)$ & $0.52(0.39-0.70)$ & $<0.0001$ & Reference & $1.03(0.88-1.21)$ & $0.91(0.77-1.07)$ & $0.89(0.75-1.05)$ & 0.068 & Reference & $0.85(0.74-0.98)$ & $0.65(0.56-0.76)$ & $0.60(0.51-0.70)$ & $<0.0001$ \\
\hline Adjusted HR/OR (95\% Cl) & Reference & $0.88(0.68-1.16)$ & $0.73(0.55-0.98)$ & $0.73(0.54-0.99)$ & 0.022 & Reference & $1.03(0.88-1.21)$ & $0.94(0.79-1.11)$ & $0.93(0.79-1.11)$ & 0.2639 & Reference & $0.96(0.81-1.13)$ & $0.81(0.68-0.97)$ & $0.83(0.70-0.99)$ & 0.008 \\
\hline
\end{tabular}


Table S5 HRs or ORs and 95\% CIs of adverse clinical outcomes at 3-month follow-up by leukocyte count, neutrophil percent, lymphocyte percent, monocyte percent, eosinophil percent, basophil percent

\begin{tabular}{|c|c|c|c|c|c|c|c|c|c|c|c|c|c|c|c|}
\hline \multirow{2}{*}{ Variable } & \multicolumn{5}{|c|}{ Death } & \multicolumn{5}{|c|}{ Stroke recurrence } & \multicolumn{5}{|c|}{$3 \leq \mathrm{mRS} \leq 6$} \\
\hline & Q1 & Q2 & Q3 & Q4 & $\mathrm{P}$ & Q1 & Q2 & Q3 & Q4 & $\mathrm{P}$ & Q1 & Q2 & Q3 & Q4 & $P$ \\
\hline Leukocyte count, n (\%) & $28(0.79)$ & $34(0.96)$ & $46(1.30)$ & $96(2.70)$ & & $187(5.26)$ & $180(5.10)$ & $215(6.07)$ & $290(8.17)$ & & $360(10.14)$ & 376 (10.65) & $474(13.38)$ & $705(19.86)$ & \\
\hline Crude HR/OR $(95 \% \mathrm{Cl})$ & Reference & $1.20(0.73-1.99)$ & $1.68(1.05-2.70)$ & $3.17(2.07-4.87)$ & $<0.0001$ & Reference & $0.95(0.77-1.17)$ & $1.14(0.93-1.39)$ & $1.54(1.28-1.86)$ & $<0.0001$ & Reference & $1.03(0.88-1.20)$ & $1.36(1.17-1.59)$ & $2.15(1.86-2.48)$ & $<0.0001$ \\
\hline Adjusted HR/OR (95\% Cl) & Reference & $1.33(0.80-2.22)$ & $1.75(1.08-2.85)$ & $2.79(1.78-4.39)$ & $<0.0001$ & Reference & $0.97(0.79-1.19)$ & $1.13(0.93-1.39)$ & $1.48(1.22-1.80)$ & $<0.0001$ & Reference & $1.04(0.88-1.23)$ & $1.29(1.09-1.52)$ & $1.85(1.57-2.17)$ & $<0.0001$ \\
\hline Neutrophil percent, n (\%) & $17(0.48)$ & $30(0.85)$ & $41(1.16)$ & $116(3.27)$ & & $179(5.05)$ & $165(4.66)$ & $216(6.09)$ & $312(8.81)$ & & $248(7.00)$ & $357(10.07)$ & $493(13.91)$ & $817(23.06)$ & \\
\hline Crude HR/OR $(95 \%$ Cl) & Reference & $1.60(0.88-2.91)$ & $2.21(1.25-3.91)$ & $5.96(3.56-9.97)$ & $<0.0001$ & Reference & $0.89(0.72-1.10)$ & $1.15(0.94-1.41)$ & $1.68(1.39-2.03)$ & $<0.0001$ & Reference & $1.43(1.21-1.70)$ & $2.09(1.77-2.46)$ & $3.81(3.26-4.46)$ & $<0.0001$ \\
\hline Adjusted HR/OR (95\% Cl) & Reference & $1.53(0.83-2.80)$ & $1.81(1.02-3.22)$ & $3.22(1.88-5.51)$ & $<0.0001$ & Reference & $0.88(0.71-1.09)$ & $1.10(0.90-1.35)$ & $1.50(1.23-1.82)$ & $<0.0001$ & Reference & $1.34(1.11-1.62)$ & $1.69(1.42-2.02)$ & $2.36(1.99-2.81)$ & $<0.0001$ \\
\hline Crude HR/OR (95\% Cl) & Reference & $0.34(0.23-0.49)$ & $0.31(0.21-0.47)$ & $0.16(0.10-0.28)$ & $<0.0001$ & Reference & $0.64(0.53-0.76)$ & $0.54(0.45-0.66)$ & $0.59(0.49-0.72)$ & $<0.0001$ & Reference & $0.54(0.48-0.62)$ & $0.37(0.32-0.42)$ & $0.26(0.22-0.31)$ & $<0.0001$ \\
\hline Adjusted HR/OR (95\% Cl) & Reference & $0.53(0.36-0.78)$ & $0.57(0.38-0.87)$ & $0.31(0.18-0.54)$ & $<0.0001$ & Reference & $0.68(0.57-0.82)$ & $0.61(0.50-0.74)$ & $0.66(0.54-0.80)$ & $<0.0001$ & Reference & $0.72(0.63-0.84)$ & $0.55(0.47-0.65)$ & $0.41(0.35-0.49)$ & $<0.0001$ \\
\hline Monocyte percent, n (\%) & $57(1.61)$ & $52(1.47)$ & $51(1.44)$ & $44(1.24)$ & & $248(7.00)$ & $229(6.46)$ & $217(6.12)$ & $178(5.02)$ & & $527(14.87)$ & $463(13.06)$ & $453(12.79)$ & 472 (13.32) & \\
\hline Crude HR/OR (95\% Cl) & Reference & $0.81(0.55-1.20)$ & $0.77(0.52-1.13)$ & $0.60(0.40-0.92)$ & 0.0193 & Reference & $0.94(0.78-1.14)$ & $0.91(0.75-1.10)$ & $0.73(0.59-0.90)$ & 0.0037 & Reference & $0.79(0.68-0.91)$ & $0.76(0.65-0.87)$ & $0.74(0.63-0.85)$ & 0.0542 \\
\hline Adjusted HR/OR (95\% Cl) & Reference & $0.84(0.56-1.25)$ & $0.81(0.54-1.22)$ & $0.56(0.36-0.87)$ & 0.0124 & Reference & $0.96(0.80-1.16)$ & $0.94(0.77-1.14)$ & $0.75(0.60-0.92)$ & 0.01 & Reference & $0.82(0.70-0.96)$ & $0.83(0.71-0.98)$ & $0.77(0.65-0.91)$ & 0.0869 \\
\hline Eosinophil percent, $\mathrm{n}(\%)$ & $98(2.77)$ & $37(1.04)$ & $32(0.91)$ & $37(1.04)$ & & $283(7.99)$ & $205(5.77)$ & $192(5.43)$ & $192(5.41)$ & & $719(20.29)$ & $450(12.68)$ & 387 (10.95) & 359 (10.12) & \\
\hline Crude HR/OR (95\% Cl) & Reference & $0.40(0.27-0.59)$ & $0.35(0.24-0.53)$ & $0.40(0.27-0.59)$ & $<0.0001$ & Reference & $0.73(0.61-0.88)$ & $0.67(0.55-0.80)$ & $0.68(0.56-0.81)$ & $<0.0001$ & Reference & $0.59(0.51-0.67)$ & $0.49(0.42-0.56)$ & $0.45(0.39-0.52)$ & $<0.0001$ \\
\hline Basophil percent, n (\%) & $67(1.89)$ & $61(1.72)$ & $42(1.18)$ & $34(0.96)$ & & $230(6.49)$ & $230(6.50)$ & $214(6.03)$ & $198(5.59)$ & & $604(17.05)$ & $501(14.16)$ & 417 (11.74) & $393(11.09)$ & \\
\hline Crude HR/OR (95\% Cl) & Reference & $0.68(0.47-0.98)$ & $0.41(0.27-0.62)$ & $0.34(0.22-0.53)$ & $<0.0001$ & Reference & $0.92(0.75-1.12)$ & $0.85(0.69-1.05)$ & $0.81(0.65-1.00)$ & 0.038 & Reference & $0.73(0.63-0.84)$ & $0.55(0.47-0.64)$ & $0.51(0.44-0.60)$ & $<0.0001$ \\
\hline Adjusted HR/OR (95\% Cl) & Reference & $0.78(0.53-1.14)$ & $0.55(0.36-0.85)$ & $0.50(0.32-0.80)$ & 0.0011 & Reference & $0.93(0.76-1.13)$ & $0.89(0.73-1.10)$ & $0.88(0.71-1.09)$ & 0.2248 & Reference & $0.81(0.69-0.95)$ & $0.68(0.58-0.81)$ & $0.70(0.59-0.84)$ & $<0.0001$ \\
\hline
\end{tabular}

mRS, modified Rankin Scale; HR, hazard ratio; OR, odds ratio; $\mathrm{Cl}$, confidence interval. 


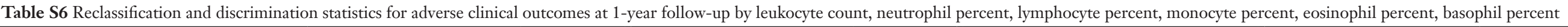

\begin{tabular}{|c|c|c|c|c|c|c|c|c|}
\hline \multirow{2}{*}{ Model } & \multicolumn{2}{|l|}{ C statistic } & \multicolumn{2}{|l|}{ IDI } & \multicolumn{2}{|c|}{ NRI (continuous) } & \multicolumn{2}{|c|}{$\mathrm{NRI}($ categorical)* } \\
\hline & Estimate $(95 \% \mathrm{Cl})$ & $P$ value & Estimate (95\% Cl), \% & $P$ value & Estimate (95\% Cl), \% & $P$ value & Estimate (95\% Cl), \% & $P$ value \\
\hline \multicolumn{9}{|l|}{ Death } \\
\hline Conventional model ${ }^{\dagger}$ & $0.804(0.783-0.826)$ & & Reference & & Reference & & Reference & \\
\hline Conventional model + leukocyte count & $0.809(0.788-0.831)$ & 0.067 & $0.008(0.004-0.012)$ & $<0.001$ & $0.203(0.109-0.298)$ & $<0.001$ & $0.024(-0.005-0.053)$ & 0.102 \\
\hline $\begin{array}{l}\text { Conventional model + neutrophil } \\
\text { percent }\end{array}$ & $0.810(0.789-0.831)$ & 0.056 & $0.006(0.004-0.009)$ & $<0.001$ & $0.292(0.199-0.385)$ & $<0.001$ & $0.029(0.0003-0.058)$ & 0.049 \\
\hline $\begin{array}{l}\text { Conventional model + lymphocyte } \\
\text { percent }\end{array}$ & $0.811(0.789-0.831)$ & 0.042 & $0.008(0.005-0.011)$ & $<0.001$ & $0.311(0.218-0.403)$ & $<0.001$ & $0.028(-0.002-0.059)$ & 0.070 \\
\hline $\begin{array}{l}\text { Conventional model + monocyte } \\
\text { percent }\end{array}$ & $0.804(0.783-0.826)$ & 0.734 & $0.0002(-0.0002-0.0006)$ & 0.233 & $0.014(-0.080-0.208)$ & 0.767 & $0.002(-0.006-0.010)$ & 0.604 \\
\hline $\begin{array}{l}\text { Conventional model + eosinophil } \\
\text { percent }\end{array}$ & $0.805(0.783-0.826)$ & 0.664 & $0.001(0.0002-0.002)$ & 0.018 & $0.123(0.034-0.213)$ & 0.010 & $-0.0004(-0.013-0.012)$ & 0.954 \\
\hline Conventional model + basophil percent & $0.804(0.783-0.826)$ & 0.762 & $0.001(-0.001-0.003)$ & 0.325 & $-0.007(-0.096-0.083)$ & 0.888 & $-0.002(-0.012-0.007)$ & 0.625 \\
\hline \multicolumn{9}{|l|}{ Stroke recurrence } \\
\hline Conventional model ${ }^{\dagger}$ & $0.601(0.585-0.617)$ & & Reference & & Reference & & Reference & \\
\hline Conventional model + leukocyte count & $0.610(0.594-0.626)$ & 0.004 & $0.002(0.0008-0.002)$ & $<0.001$ & $0.142(0.086-0.198)$ & $<0.001$ & $0.003(-0.013-0.020)$ & 0.674 \\
\hline $\begin{array}{l}\text { Conventional model + neutrophil } \\
\text { percent }\end{array}$ & $0.614(0.598-0.630)$ & $<0.001$ & $0.002(0.001-0.003)$ & $<0.001$ & $0.134(0.079-0.190)$ & $<0.001$ & $0.001(-0.018-0.020)$ & 0.879 \\
\hline $\begin{array}{l}\text { Conventional model + lymphocyte } \\
\text { percent }\end{array}$ & $0.613(0.597-0.628)$ & $<0.001$ & $0.002(0.001-0.002)$ & $<0.001$ & $0.114(0.058-0.170)$ & $<0.001$ & $0.001(-0.016-0.019)$ & 0.884 \\
\hline $\begin{array}{l}\text { Conventional model + monocyte } \\
\text { percent }\end{array}$ & $0.606(0.590-0.622)$ & 0.082 & $0.001(0.0005-0.002)$ & $<0.001$ & $0.085(0.030-0.141)$ & 0.003 & $-0.011(-0.026-0.004)$ & 0.163 \\
\hline $\begin{array}{l}\text { Conventional model + eosinophil } \\
\text { percent }\end{array}$ & $0.604(0.588-0.620)$ & 0.039 & $0.0002(-0.0001-0.0005)$ & 0.127 & $0.057(0.003-0.110)$ & 0.047 & $0.008(-0.002-0.002)$ & 0.137 \\
\hline Conventional model + basophil percent & $0.601(0.585-0.617)$ & 0.324 & $0.0007(-0.0001-0.0003)$ & 0.505 & $-0.057(-0.111-0.004)$ & 0.044 & $-0.004(-0.009-0.003)$ & 0.069 \\
\hline \multicolumn{9}{|l|}{$3 \leq \mathrm{mRS} \leq 6$} \\
\hline Conventional model ${ }^{\dagger}$ & $0.798(0.787-0.809)$ & & Reference & & Reference & & Reference & \\
\hline Conventional model + leukocyte count & $0.802(0.791-0.813)$ & $<0.001$ & $0.006(0.004-0.008)$ & $<0.001$ & $0.210(0.162-0.259)$ & $<0.001$ & $0.017(0.003-0.031)$ & 0.018 \\
\hline $\begin{array}{l}\text { Conventional model + neutrophil } \\
\text { percent }\end{array}$ & $0.804(0.793-0.815)$ & $<0.001$ & $0.007(0.005-0.010)$ & $<0.001$ & $0.256(0.208-0.305)$ & $<0.001$ & $0.026(0.010-0.041)$ & 0.002 \\
\hline $\begin{array}{l}\text { Conventional model + lymphocyte } \\
\text { percent }\end{array}$ & $0.805(0.794-0.816)$ & $<0.001$ & $0.009(0.007-0.012)$ & $<0.001$ & $0.276(0.228-0.324)$ & $<0.001$ & $0.025(0.008-0.042)$ & 0.004 \\
\hline $\begin{array}{l}\text { Conventional model + monocyte } \\
\text { percent }\end{array}$ & $0.798(0.787-0.809)$ & 0.253 & $0(0-0)$ & 0.749 & $0.004(-0.045-0.053)$ & 0.875 & $0.0009(-0.0008-0.003)$ & 0.298 \\
\hline $\begin{array}{l}\text { Conventional model + eosinophil } \\
\text { percent }\end{array}$ & $0.798(0.787-0.809)$ & 0.376 & $0.0005(-0.0001-0.001)$ & 0.079 & $0.088(0.041-0.134)$ & $<0.001$ & $0.002(-0.004-0.009)$ & 0.485 \\
\hline Conventional model + basophil percent & $0.798(0.787-0.809)$ & 0.566 & $0.0004(-0.0002-0.0003)$ & 0.693 & $-0.038(-0.086-0.009)$ & 0.122 & $0.002(-0.0007-0.005)$ & 0.134 \\
\hline
\end{tabular}

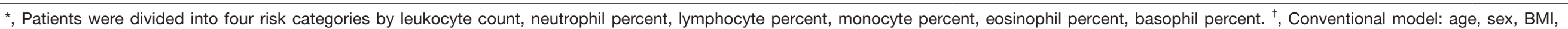

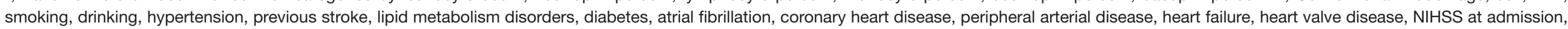

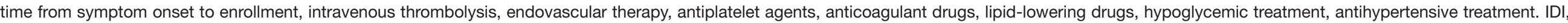
integrated discrimination improvement; NRI, net reclassification index; Cl, confidence interval; mRS, modified Rankin Scale; BMI, body mass index; NIHSS, National Institutes of Health Stroke Scale. 